\title{
Energy levels and radiative rates for transitions in Co XI
}

\author{
K. M. Aggarwal ${ }^{1}$, F. P. Keenan ${ }^{1}$, and A. Z. Msezane ${ }^{2}$ \\ 1 Astrophysics Research Centre, School of Mathematics and Physics, Queen's University, Belfast BT7 1NN, Northern Ireland, UK \\ e-mail: K.Aggarwal@qub.ac.uk \\ 2 Centre for Theoretical Studies of Physical Systems, Clark Atlanta University, Atlanta Ga 30304, USA
}

Received 5 June 2007 / Accepted 19 July 2007

\begin{abstract}
Aims. In this paper we report calculations for energy levels and radiative rates for transitions in Co XI.

Methods. The General purpose Relativistic Atomic Structure Package (GRASP) and the Flexible Atomic Code (FAC) have been adopted for the calculations of energy levels and radiative rates.

Results. Energies for the lowest 287 levels of Co XI, including those among the $\left(1 s^{2} 2 s^{2} 2 p^{6}\right) 3 s^{2} 3 p^{5}, 3 s 3 p^{6}, 3 s^{2} 3 p^{4} 3 d, 3 s 3 p^{5} 3 d$, $3 s^{2} 3 p^{3} 3 d^{2}$, and $3 s^{2} 3 p^{4} 4 s$ configurations, are reported. Additionally, radiative rates and oscillator strengths are reported for all electric dipole (E1) transitions with $f \geq 10^{-5}$ among these levels, and similar results for magnetic dipole (M1), electric quadrupole (E2), and magnetic quadrupole (M2) transitions are reported only for those with $f \geq 10^{-8}$. Comparisons are made with the available results in the literature, and the accuracy of the present data is assessed. Finally, lifetimes for all excited levels are also listed, although measurements are presently available for only one of these.
\end{abstract}

Key words. atomic data - atomic processes

\section{Introduction}

Iron group elements $(\mathrm{Sc}-\mathrm{Zn})$ are becoming increasing important in astrophysical plasmas, because many of their emission lines are frequently observed from their different ionisation stages. These observations provide a wealth of data about the plasma characteristics, such as: temperature, density and chemical composition. Additionally, iron group elements are often impurities in fusion reactors, and to estimate the power loss from the impurities, atomic data (namely energy levels, oscillator strengths or radiative decay rates, collision strengths, and excitation rates) are required for many ions. Since there is paucity of measured parameters, one must depend on theoretical results. Therefore, in the recent past we have reported atomic parameters for many ions of Fe (IX-XXVI, see Aggarwal \& Keenan 2006, and references therein) and Ni (XIII-XIX, see Aggarwal et al. 2007, and references therein). In this paper we focus our attention on Cl-like Co XI.

Cobalt, being an odd atomic number $(Z)$ element, is comparatively less abundant in astrophysical plasmas than the even- $Z$ elements of the iron group, such as $\mathrm{Cr}, \mathrm{Fe}$ and $\mathrm{Ni}$. Nevertheless, several emission lines from this element have been observed in solar plasmas by Thomas \& Neupert (1994), and identified in the spectra from theta-pinch plasmas by Fawcett \& Hayes (1972), Fawcett et al. (1972), and Fawcett $\&$ Hatter (1980). Additionally, some of the lines from Cllike Co XI, particularly in the 65-340 $\AA$ wavelength range, are listed in the Atomic Line List (v2.04) of Peter van Hoof at http://www.pa.uky.edu/ peter/atomic/. Atomic data

^ Tables 1 and 3-6 are only available in electronic form at the CDS via anonymous ftp to

cdsarc.u-strasbg.fr $(130.79 .128 .5)$ or via

http://cdsweb.u-strasbg.fr/cgi-bin/qcat?J/A+A/473/995 are required for the generation of reliable synthetic spectra, and a specific line list helps in the proper identification of observed wavelengths. Additionally, the lifetime for one of the levels of Co XI has already been measured in laboratory plasmas by Träbert et al. (2004, 2006), and hence it provides a further impetus for theoretical work.

Due to the importance of Cl-like ions, many workers have performed calculations for atomic parameters adopting a variety of methods. Particularly noteworthy are the calculations for Co XI by Huang et al. (1983), Biémont \& Träbert (2000), and Berrington et al. (2001). Huang et al. performed relativistic calculations among the lowest 31 levels of the $3 \mathrm{~s}^{2} 3 \mathrm{p}^{5}, 3 \mathrm{~s} 3 \mathrm{p}^{6}$ and $3 \mathrm{~s}^{2} 3 \mathrm{p}^{4} 3 \mathrm{~d}$ configurations. They adopted the multi-configuration Dirac-Fock (MCDF) method, but only reported radiative rates $(A$ values) from the levels of the $3 \mathrm{~s}^{2} 3 \mathrm{p}^{5}$ ground configuration to higher excited levels. Furthermore, they included only limited CI (configuration interaction) in the construction of wavefunctions, which was found to be inadequate by subsequent workers. This deficiency was addressed to a large extent by Biémont \& Träbert and Berrington et al., but their calculations focused only on the lowest three levels, namely $3 s^{2} 3 p^{5}{ }^{2} \mathrm{P}_{1 / 2,3 / 2}^{\circ}$ and $3 \mathrm{~s} 3 \mathrm{p}^{6}$ ${ }^{2} S_{1 / 2}$. Therefore, in a more recent paper Verma et al. (2005) have reported energy levels and $A$ - values for many transitions. They have adopted the CIV3 program of Hibbert (1975a), and have included extensive $\mathrm{CI}$ as well as one-body relativistic operators in the generation of wavefunctions. Furthermore, to improve the accuracy of their energy levels, they have adjusted the Hamiltonian in accordance with the experimental energies. However, experimental values are available for only 17 of the 65 energy levels $(25 \%)$ of their calculations. This gives scope for improvement, particularly for the higher levels of their calculations for which no measurements are available. Additionally, there is a wide gap of $\sim 5$ Ryd between the energies of the 
$3 s^{2} 3 p^{4}\left({ }^{3} \mathrm{P}\right) 3 d^{2} \mathrm{D}_{3 / 2}$ and $3 s^{2} 3 p^{4}\left({ }^{3} \mathrm{P}\right) 4 s^{4} \mathrm{P}_{5 / 2}$ levels - see Table 4 of Verma et al. or Table 1 in the present paper. Many levels of the $3 s 3 p^{5} 3 d, 3 p^{3} 3 d^{2}$ and $3 s 3 p^{4} 3 d^{2}$ configurations lie in this energy interval, which have been excluded, although they have been included in the expansion of the wavefunctions. Therefore, we extend their calculations by including the missing levels of these configurations, because a larger model is desirable in plasma modelling, the importance of which has been emphasized and demonstrated by Liedahl (2000). Finally, they calculated the $A$ values for the electric dipole transitions alone, and reported results only from the levels of the $3 \mathrm{~s}^{2} 3 \mathrm{p}^{5}$ ground configuration to higher excited levels. However in modelling and diagnostic applications, corresponding results are required for all transitions, as well as for other types of transitions, as emphasized and demonstrated by Del Zanna et al. (2004). Therefore, we are reporting radiative rates for four types of transitions, namely electric dipole (E1), electric quadrupole (E2), magnetic dipole (M1), and magnetic quadrupole (M2), among the lowest $287 \mathrm{lev}$ els of Co XI, although calculations have been performed for up to 19401 levels. Finally, we also report results of lifetimes for all levels, although measurements are so far available for only one.

For our calculations, we have adopted the GRASP (General purpose Relativistic Atomic Structure Package) code, which was originally developed as GRASP0 by Grant et al. (1980). A revised and modified version of this code was published as GRASP1 by Dyall et al. (1989), which has been further updated by Dr. P. H. Norrington (http://www . am . qub . ac. uk/DARC/). This is a fully relativistic code, and is based on the $j j$ coupling scheme. Further relativistic corrections arising from the Breit interaction and QED effects have also been included. Additionally, we have used the option of extended average level (EAL), in which a weighted (proportional to $2 j+1$ ) trace of the Hamiltonian matrix is minimized. This produces a compromise set of orbitals describing closely lying states with moderate accuracy.

\section{Energy levels}

The $\left(1 s^{2} 2 s^{2} 2 p^{6}\right) 3 s^{2} 3 p^{5}, 3 s 3 p^{6}$ and $3 s^{2} 3 p^{4} 3 d$ configurations of Co XI give rise to energetically lowest 31 levels, listed in Table 1 . These levels cover an energy range of up to $\sim 6$ Ryd. Beyond these lie a range of energy levels from the $3 s^{2} 3 p^{3} 3 d^{2}, 3 s 3 p^{5} 3 d$, $3 s^{2} 3 p^{2} 3 d^{3}, 3 s 3 p^{4} 3 d^{2}, 3 s 3 p^{3} 3 d^{3}, 3 p^{6} 3 d$, and $3 p^{5} 3 d^{2}$ configurations. These seven configurations give rise to 1361 levels and almost all are intermixed as seen in Table 1 . Therefore, a calculation involving the 1392 levels from the above listed 10 configurations becomes necessary in order to achieve a reasonable accuracy. Similarly, the $3 s^{2} 3 p^{4} 4 \ell$ configurations give rise to 87 levels, which are also intermixed with the levels of the above configurations. Levels from two of these configurations $\left(3 \mathrm{~s}^{2} 3 \mathrm{p}^{4} 4 \mathrm{~s}\right.$ and $3 s^{2} 3 p^{4} 4 d$ ) were included by Verma et al. (2005). However, even this large calculation is not fully sufficient, as 1129 levels of the additional 13 configurations, namely $3 \mathrm{~s}^{2} 3 \mathrm{p}^{3} 3 \mathrm{~d} 4 \ell$, $3 \mathrm{~s} 3 \mathrm{p}^{5} 4 \ell$ and $3 \mathrm{~s}^{2} 3 \mathrm{p}^{4} 5 \ell$, closely interact with the 1479 levels listed above. CI among all these levels/configurations improves the accuracy of the energy levels, and subsequently of the radiative rates. Therefore, using the GRASP code we have performed a series of calculations, but will focus only on three, namely: GRASP1, which includes 1392 levels from the lowest 10 configurations $\left(3 \mathrm{~s}^{2} 3 \mathrm{p}^{5}, 3 \mathrm{~s} 3 \mathrm{p}^{6}, 3 \mathrm{~s}^{2} 3 \mathrm{p}^{4} 3 \mathrm{~d}, 3 \mathrm{~s}^{2} 3 \mathrm{p}^{3} 3 \mathrm{~d}^{2}, 3 \mathrm{~s} 3 \mathrm{p}^{5} 3 \mathrm{~d}\right.$, $3 s^{2} 3 p^{2} 3 d^{3}, 3 s 3 p^{4} 3 d^{2}, 3 s 3 p^{3} 3 d^{3}, 3 p^{6} 3 d$, and $3 p^{5} 3 d^{2}$ ); GRASP2, which includes additional 87 levels from the $3 \mathrm{~s}^{2} 3 \mathrm{p}^{4} 4 \ell$ configurations; and GRASP3, which includes a total of 2608 levels from all the 27 configurations listed above.
Since cobalt is a moderately heavy element, both relativistic effects and CI are important in the accurate determination of energy levels, as already discussed and demonstrated by Aggarwal et al. (2007) for Fe XV, Co XVI and Ni XVII. Furthermore, Verma et al. (2005) had already included a larger CI among 52 configurations listed in their Table 3 . Therefore, it has become necessary to assess the effect of a larger CI in order to have a confidence in the accuracy of the theoretical results. To achieve this aim, we have performed additional calculations from the Flexible Atomic Code (FAC) of Gu (2003), which is available from the website http://kipac-tree.stanford.edu/fac. This is a fully relativistic code as is GRASP, and gives comparable results, which helps in further assessing the accuracy. From this code we have performed a series of calculations, but focus on only three: FAC1, which includes the 2608 levels as in GRASP3 mentioned above; FAC2, which includes 5709 levels from all possible configurations from the $n=3$ orbitals in addition to $3 \mathrm{~s}^{2} 3 \mathrm{p}^{4} 4 \ell, 3 \mathrm{~s}^{2} 3 \mathrm{p}^{4} 5 \ell, 3 \mathrm{~s}^{2} 3 \mathrm{p}^{3} 3 \mathrm{~d} 4 \ell$, and $3 \mathrm{~s} 3 \mathrm{p}^{5} 4 \ell$; and FAC 3 , which includes a total of 19401 levels, the additional $13692 \mathrm{lev}-$ els coming from the $3 \mathrm{~s}^{2} 3 \mathrm{p}^{3} 4 \ell^{2}, 3 \mathrm{p}^{5} 4 \ell^{2}, 3 \mathrm{p}^{6} 4 \ell, 3 \mathrm{p}^{6} 5 \ell, 3 \mathrm{p}^{5} 3 \mathrm{~d} 4 \ell$, $3 \mathrm{~s} 3 \mathrm{p}^{4} 3 \mathrm{~d} 4 \ell, 3 \mathrm{p}^{4} 3 \mathrm{~d}^{2} 4 \ell, 3 \mathrm{~s} 3 \mathrm{p}^{5} 5 \ell, 3 \mathrm{~s}^{2} 3 \mathrm{p}^{3} 4 \ell 5 \ell$, and $3 \mathrm{~s}^{2} 3 \mathrm{p}^{3} 5 \ell^{2}$ configurations.

For conciseness, in Table 1 we report our energies for the lowest 287 levels alone, which include all levels from the $3 s^{2} 3 p^{5}$ (2), $3 s 3 p^{6} \quad(1), 3 s^{2} 3 p^{4} 3 d \quad(28), 3 s 3 p^{5} 3 d \quad(23)$, $3 s^{2} 3 p^{3} 3 d^{2}(144)$ and $3 s^{2} 3 p^{4} 4 s$ (8) configurations. However, a complete set of results from any of our above listed six specific calculations, i.e. GRASP1, GRASP2, GRASP3, FAC1, FAC2 and FAC3, can be obtained on request from KMA (K.Aggarwal@qub.ac.uk). The listed energies are from our GRASP3 and FAC1 calculations. Furthermore, results from the GRASP code are listed from the calculations performed with and without including Breit and QED effects. The inclusion of Breit and QED effects lowers the energies by up to a maximum of 0.2 Ryd $(\leq .25 \%)$ - see for example, levels 18-24 and 48-58. Similarly, our energy levels from both the GRASP and FAC calculations agree closely and the differences are within $0.04 \mathrm{Ryd}(\leq 0.04 \%)$ - see, for example, levels $27-28$ and 186-187. However, the level orderings in the two calculations are slightly different in a few instances, such as for levels: $64 / 65\left(3 \mathrm{~s}^{2} 3 \mathrm{p}^{3}\left({ }^{4} \mathrm{~S}\right) 3 \mathrm{~d}^{2}{ }^{4} \mathrm{D}_{3 / 2,5 / 2}^{\circ}\right), 81 / 83\left(3 \mathrm{~s}^{2} 3 \mathrm{p}^{3}\left({ }^{2} \mathrm{P}\right) 3 \mathrm{~d}^{2}{ }^{2} \mathrm{D}_{3 / 2}^{\circ}\right.$ and $\left.3 \mathrm{~s}^{2} 3 \mathrm{p}^{3}\left({ }^{2} \mathrm{D}\right) 3 \mathrm{~d}^{2}{ }^{4} \mathrm{H}_{13 / 2}^{\circ}\right)$ and $104 / 105\left(3 \mathrm{~s}^{2} 3 \mathrm{p}^{3}\left({ }^{2} \mathrm{P}\right) 3 \mathrm{~d}^{2}{ }^{2} \mathrm{D}_{5 / 2}^{\circ}\right.$ and $\left.3 s^{2} 3 p^{3}\left({ }^{2} D\right) 3 d^{2}{ }^{2} I_{13 / 2}^{\circ}\right)$. Most of these levels have energies very close to each other, and the level orderings from the FAC calculations are generally in better agreement with our GRASP results performed without the inclusion of Breit and QED effects.

In Table 2a we compare our energies for the lowest 31 levels of Table 1, which are obtained from the GRASP1, GRASP2 and GRASP3 calculations from the GRASP code, and FAC1, FAC2 and FAC 3 from the FAC code. All these calculations are with increasing amount of $\mathrm{CI}$, but the level orderings are the same for the lowest 31 levels listed here. Furthermore, the maximum difference between any two calculations is $\leq 0.04 \mathrm{Ryd}$, or equivalently, better than $1 \%$ for any level. This clearly indicates that for the lowest 31 levels considered here, an elaborate CI does not improve the energy levels. However, a larger CI as adopted in our GRASP3 calculations compared to GRASP1 slightly improves energies for the higher levels, as can also be noticed for levels $27-31$ in Table 2a. A further increase of CI, as adopted in our calculations from FAC, yields results within $0.2 \mathrm{Ryd}$ for all levels listed in Table 1. Therefore, we may state with confidence that more CI than is included in our GRASP3 and FAC1 calculations is of no significant advantage as far as the lowest 
Table 2. a) Comparison of energy levels (in Ryd) of Co XI.

\begin{tabular}{|c|c|c|c|c|c|c|c|c|}
\hline Index & Configuration & Level & GRASP1 & GRASP2 & GRASP3 & FAC1 & FAC2 & $\overline{\text { FAC3 }}$ \\
\hline 1 & $3 s^{2} 3 p^{5}$ & ${ }^{2} \mathrm{P}_{3 / 2}^{\circ}$ & 0.0000 & 0.0000 & 0.0000 & 0.0000 & 0.0000 & 0.0000 \\
\hline 2 & $3 s^{2} 3 p^{5}$ & ${ }^{2} \mathrm{P}_{1 / 2}^{\circ}$ & 0.1760 & 0.1757 & 0.1755 & 0.1750 & 0.1751 & 0.1752 \\
\hline 3 & $3 s 3 p^{6}$ & ${ }^{2} \mathrm{~S}_{1 / 2}$ & 2.8449 & 2.8475 & 2.8459 & 2.8541 & 2.8523 & 2.8593 \\
\hline 4 & $3 s^{2} 3 p^{4}\left({ }^{3} P\right) 3 d$ & ${ }^{4} \mathrm{D}_{5 / 2}$ & 3.8468 & 3.8509 & 3.8405 & 3.8419 & 3.8227 & 3.8387 \\
\hline 5 & $3 \mathrm{~s}^{2} 3 \mathrm{p}^{4}\left({ }^{3} \mathrm{P}\right) 3 \mathrm{~d}$ & ${ }^{4} \mathrm{D}_{7 / 2}$ & 3.8483 & 3.8524 & 3.8419 & 3.8434 & 3.8241 & 3.8401 \\
\hline 6 & $3 s^{2} 3 p^{4}\left({ }^{3} P\right) 3 d$ & ${ }^{4} \mathrm{D}_{3 / 2}$ & 3.8604 & 3.8645 & 3.8541 & 3.8555 & 3.8363 & 3.8524 \\
\hline 7 & $3 \mathrm{~s}^{2} 3 \mathrm{p}^{4}\left({ }^{3} \mathrm{P}\right) 3 \mathrm{~d}$ & $\begin{array}{l}{ }^{4} \mathrm{D}_{1 / 2} \\
1 / 2\end{array}$ & 3.8786 & 3.8827 & 3.8722 & 3.8737 & 3.8543 & 3.8703 \\
\hline 8 & $3 s^{2} 3 p^{4}\left({ }^{3} P\right) 3 d$ & ${ }^{4} \mathrm{~F}_{9 / 2}$ & 4.1548 & 4.1604 & 4.1459 & 4.1448 & 4.1265 & 4.1412 \\
\hline 9 & $3 s^{2} 3 p^{4}\left({ }^{1} D\right) 3 d$ & ${ }^{2} \mathrm{P}_{1 / 2}$ & 4.1790 & 4.1839 & 4.1685 & 4.1688 & 4.1462 & 4.1635 \\
\hline 10 & $3 s^{2} 3 p^{4}\left({ }^{3} P\right) 3 d$ & ${ }^{4} \mathrm{~F}_{7 / 2}$ & 4.2115 & 4.2169 & 4.2024 & 4.2010 & 4.1828 & 4.1975 \\
\hline 11 & $3 s^{2} 3 p^{4}\left({ }^{3} P\right) 3 d$ & ${ }^{4} \mathrm{~F}_{5 / 2}$ & 4.2553 & 4.2607 & 4.2462 & 4.2451 & 4.2269 & 4.2415 \\
\hline 12 & $3 s^{2} 3 p^{4}\left({ }^{1} D\right) 3 d$ & ${ }^{2} \mathrm{P}_{3 / 2}$ & 4.2660 & 4.2712 & 4.2562 & 4.2555 & 4.2345 & 4.2513 \\
\hline 13 & $3 s^{2} 3 p^{4}\left({ }^{3} P\right) 3 d$ & ${ }^{4} \mathrm{~F}_{3 / 2}$ & 4.2706 & 4.2757 & 4.2612 & 4.2602 & 4.2415 & 4.2568 \\
\hline 14 & $3 s^{2} 3 p^{4}\left({ }^{1} D\right) 3 d$ & ${ }^{2} \mathrm{D}_{3 / 2}$ & 4.3234 & 4.3283 & 4.3149 & 4.3126 & 4.2939 & 4.3099 \\
\hline 15 & $3 s^{2} 3 p^{4}\left({ }^{3} P\right) 3 d$ & ${ }^{4} \mathrm{P}_{1 / 2}$ & 4.3266 & 4.3323 & 4.3163 & 4.3149 & 4.2943 & 4.3111 \\
\hline 16 & $3 s^{2} 3 p^{4}\left({ }^{3} P\right) 3 d$ & ${ }^{4} \mathrm{P}_{3 / 2}$ & 4.3857 & 4.3910 & 4.3750 & 4.3737 & 4.3527 & 4.3689 \\
\hline 17 & $3 s^{2} 3 p^{4}\left({ }^{3} P\right) 3 d$ & ${ }^{2} \mathrm{~F}_{7 / 2}$ & 4.4022 & 4.4081 & 4.3918 & 4.3887 & 4.3693 & 4.3847 \\
\hline 18 & $3 s^{2} 3 p^{4}\left({ }^{3} P\right) 3 d$ & ${ }^{4} \mathrm{P}_{5 / 2}$ & 4.4027 & 4.4078 & 4.3933 & 4.3911 & 4.3723 & 4.3886 \\
\hline 19 & $3 s^{2} 3 p^{4}\left({ }^{1} D\right) 3 d$ & ${ }^{2} \mathrm{D}_{5 / 2}$ & 4.4322 & 4.4373 & 4.4222 & 4.4201 & 4.4001 & 4.4168 \\
\hline 20 & $3 s^{2} 3 p^{4}\left({ }^{1} D\right) 3 d$ & ${ }^{2} \mathrm{G}_{9 / 2}$ & 4.5116 & 4.5172 & 4.5019 & 4.4983 & 4.4804 & 4.4959 \\
\hline 21 & $3 s^{2} 3 p^{4}\left({ }^{1} D\right) 3 d$ & ${ }^{2} \mathrm{G}_{7 / 2}$ & 4.5147 & 4.5204 & 4.5044 & 4.5010 & 4.4821 & 4.4977 \\
\hline 22 & $3 s^{2} 3 p^{4}\left({ }^{3} P\right) 3 d$ & ${ }^{2} \mathrm{~F}_{5 / 2}$ & 4.5490 & 4.5550 & 4.5374 & 4.5344 & 4.5140 & 4.5294 \\
\hline 23 & $3 s^{2} 3 p^{4}\left({ }^{1} D\right) 3 d$ & ${ }^{2} \mathrm{~F}_{5 / 2}$ & 4.8289 & 4.8355 & 4.8161 & 4.8109 & 4.7887 & 4.8045 \\
\hline 24 & $3 \mathrm{~s}^{2} 3 \mathrm{p}^{4}\left({ }^{1} \mathrm{D}\right) 3 \mathrm{~d}$ & ${ }^{2} \mathrm{~F}_{7 / 2}$ & 4.8740 & 4.8806 & 4.8611 & 4.8557 & 4.8337 & 4.8494 \\
\hline 25 & $3 s^{2} 3 p^{4}\left({ }^{1} S\right) 3 d$ & ${ }^{2} \mathrm{D}_{3 / 2}$ & 5.1074 & 5.1141 & 5.0998 & 5.0946 & 5.0840 & 5.0994 \\
\hline 26 & $3 s^{2} 3 p^{4}\left({ }^{1} S\right) 3 d$ & ${ }^{2} \mathrm{D}_{5 / 2}$ & 5.1577 & 5.1644 & 5.1505 & 5.1453 & 5.1357 & 5.1509 \\
\hline 27 & $3 s^{2} 3 p^{4}\left({ }^{1} D\right) 3 d$ & ${ }^{2} \mathrm{~S}_{1 / 2}$ & 5.4116 & 5.4183 & 5.4187 & 5.4013 & 5.3777 & 5.3916 \\
\hline 28 & $3 s^{2} 3 p^{4}\left({ }^{3} P\right) 3 d$ & ${ }^{2} \mathrm{P}_{3 / 2}$ & 5.6772 & 5.6800 & 5.6588 & 5.6421 & 5.6355 & 5.6512 \\
\hline 29 & $3 s^{2} 3 p^{4}\left({ }^{3} P\right) 3 d$ & ${ }^{2} \mathrm{P}_{1 / 2}$ & 5.7417 & 5.7443 & 5.7228 & 5.7057 & 5.6998 & 5.7157 \\
\hline 30 & $3 s^{2} 3 p^{4}\left({ }^{3} P\right) 3 d$ & ${ }^{2} \mathrm{D}_{5 / 2}$ & 5.7476 & 5.7530 & 5.7391 & 5.7205 & 5.7079 & 5.7221 \\
\hline 31 & $3 s^{2} 3 p^{4}\left({ }^{3} P\right) 3 d$ & ${ }^{2} \mathrm{D}_{3 / 2}$ & 5.8973 & 5.9027 & 5.8884 & 5.8698 & 5.8579 & 5.8721 \\
\hline
\end{tabular}

GRASP1: present calculations from the GRASP code with 1392 levels. GRASP2: present calculations from the GRASP code with 1479 levels. GRASP3: present calculations from the GRASP code with 2608 levels. FAC1: present calculations from the FAC code with 2608 levels. FAC2: present calculations from the FAC code with 5709 levels. FAC3: present calculations from the FAC code with 19401 levels.

287 levels are concerned. This is the main reason that in Table 1, or in subsequent tables for radiative rates, we have preferred to include results from the above named two (GRASP3 and FAC1) calculations alone.

In Table $2 b$ we compare our energies from the GRASP (GRASP3) and FAC (FAC1) calculations for the lowest 31 levels with the corresponding results of Verma et al. (2005) obtained from the CIV3 code of Hibbert (1975a). Also included in this table are the experimental energies compiled by the National Institute for Standards and Technology (NIST), which are available at their website: http://physics.nist.gov/PhysRefData, and the unpublished results of Irimia \& Froese-Fischer (2003), which have been obtained from their multi-configuration Hartree-Fock (MCHF) code, and are available at their website: http://www . vuse. vanderbilt . edu/ cff/mchf_ collection/. The experimental energies are available for only a few levels as can be seen in Table $2 b$, and the MCHF results are available for all levels except those with $J=7 / 2$ and 9/2. However, the level orderings are the same in theory (except from CIV3) and experiment, and the differences in magnitude are within 0.15 Ryd. Furthermore, the MCHF results of Irimia $\&$ Froese-Fischer are slightly closer to the experimental values, especially for the higher levels, but their energy for the $3 \mathrm{~s}^{2} 3 \mathrm{p}^{5}{ }^{2} \mathrm{P}_{1 / 2}^{\circ}$ level (2) is lower by $10 \%$. Similarly, the cIV3 results of Verma et al. are in perfect agreement with the experimental values, because they have adjusted their Hamiltonian accordingly, whereas other workers have not. However, as stated earlier, the experimental energies are available for only a few levels, and therefore we focus our attention on those levels for which (to our knowledge) no measurements are available.

A closer look at Table $2 b$ clearly reveals that the energy levels of Verma et al. (2005) not only differ in magnitude but also in orderings. Differences of up to $5 \%$ are observed for many levels, such as $\left(3 \mathrm{~s}^{2} 3 \mathrm{p}^{4}\left({ }^{3} \mathrm{P}\right) 3 \mathrm{~d}\right){ }^{4} \mathrm{D}_{5 / 2,7 / 2,3 / 2,1 / 2}(4-7)$ and $\left(3 s^{2} 3 p^{4}\left({ }^{3} P\right) 3 d\right)^{4} F_{7 / 2,5 / 2}(10-11)$. Similarly, their level orderings in many instances, such as for $\left.3 s^{2} 3 p^{4}\left({ }^{3} \mathrm{P}\right) 3 \mathrm{~d}\right){ }^{4} \mathrm{D}_{5 / 2,7 / 2,3 / 2,1 / 2}$, $\left.3 \mathrm{~s}^{2} 3 \mathrm{p}^{4}\left({ }^{1} \mathrm{D}\right) 3 \mathrm{~d}\right){ }^{2} \mathrm{D}_{3 / 2,5 / 2}$ and $\left.3 \mathrm{~s}^{2} 3 \mathrm{p}^{4}\left({ }^{3} \mathrm{P}\right) 3 \mathrm{~d}\right){ }^{2} \mathrm{~F}_{7 / 2,5 / 2}$ levels, disagree with all other calculations listed in Tables $2 \mathrm{a}, \mathrm{b}$. Furthermore, they have not only misidentified the orderings of the $3 s^{2} 3 p^{4}\left({ }^{1} \mathrm{D}\right) 3 \mathrm{~d}^{2} \mathrm{P}_{1 / 2,3 / 2}(9$ and 12$)$ and $3 \mathrm{~s}^{2} 3 \mathrm{p}^{4}\left({ }^{3} \mathrm{P}\right) 3 \mathrm{~d}^{2} \mathrm{P}_{3 / 2,1 / 2}$ (28 and 29) levels, but have also erroneously reproduced the orderings of NIST, as the correct listings are shown in Table $2 \mathrm{~b}$. Their misidentification of the levels may be due to the fact that these are highly mixed, as can be seen by their percentage 
Table 2. b) Comparison of energy levels (in Ryd) of Co XI.

\begin{tabular}{|c|c|c|c|c|c|c|c|c|}
\hline Index & Configuration & Level & NIST & GRASP & FAC & CIV3 & MCHF & $\overline{\text { Percentage contributions }}^{a}$ \\
\hline 1 & $3 s^{2} 3 p^{5}$ & ${ }^{2} \mathrm{P}_{3 / 2}^{\circ}$ & 0.00000 & 0.00000 & 0.00000 & 0.00000 & 0.0000 & $96(1)$ \\
\hline 2 & $3 s^{2} 3 p^{5}$ & ${ }^{2} \mathrm{P}_{1 / 2}^{\circ / 2}$ & 0.17628 & 0.17551 & 0.17496 & 0.17573 & 0.1600 & $96(2)$ \\
\hline 3 & $3 \mathrm{~s} 3 \mathrm{p}^{6}$ & ${ }^{2} \mathrm{~S}_{1 / 2}$ & 2.85801 & 2.84593 & 2.85414 & 2.85857 & 2.8419 & $71(3), 25(27)$ \\
\hline 4 & $3 s^{2} 3 p^{4}\left({ }^{3} P\right) 3 d$ & ${ }^{4} \mathrm{D}_{5 / 2}$ & & 3.84049 & 3.84188 & 4.01045 & 3.8461 & $92(4)$ \\
\hline 5 & $3 s^{2} 3 p^{4}\left({ }^{3} P\right) 3 d$ & ${ }^{4} \mathrm{D}_{7 / 2}$ & & 3.84188 & 3.84336 & 4.03250 & & $94(5)$ \\
\hline 6 & $3 s^{2} 3 p^{4}\left({ }^{3} P\right) 3 d$ & ${ }^{4} \mathrm{D}_{3 / 2}$ & & 3.85414 & 3.85554 & 4.00532 & 3.8574 & $91(6)$ \\
\hline 7 & $3 s^{2} 3 p^{4}\left({ }^{3} P\right) 3 d$ & ${ }^{4} \mathrm{D}_{1 / 2}$ & & 3.87218 & 3.87373 & 3.93392 & 3.8733 & $93(7)$ \\
\hline 8 & $3 s^{2} 3 p^{4}\left({ }^{3} P\right) 3 d$ & ${ }^{4} \mathrm{~F}_{9 / 2}$ & & 4.14588 & 4.14479 & 4.32742 & & $91(8)$ \\
\hline 9 & $3 s^{2} 3 p^{4}\left({ }^{1} D\right) 3 d$ & ${ }^{2} \mathrm{P}_{1 / 2}$ & & 4.16855 & 4.16876 & 5.58684 & 4.1531 & $49(9), 41(29)$ \\
\hline 10 & $3 s^{2} 3 p^{4}\left({ }^{3} P\right) 3 d$ & ${ }^{4} \mathrm{~F}_{7 / 2}$ & & 4.20240 & 4.20105 & 4.38430 & & $86(10)$ \\
\hline 11 & $3 s^{2} 3 p^{4}\left({ }^{3} P\right) 3 d$ & ${ }^{4} \mathrm{~F}_{5 / 2}$ & & 4.24622 & 4.24506 & 4.42304 & 4.2263 & $94(11)$ \\
\hline 12 & $3 s^{2} 3 p^{4}\left({ }^{1} D\right) 3 d$ & ${ }^{2} \mathrm{P}_{3 / 2}$ & & 4.25616 & 4.25552 & 5.51952 & 4.2363 & $30(12), 28(13), 23(28)$ \\
\hline 13 & $3 s^{2} 3 p^{4}\left({ }^{3} P\right) 3 d$ & ${ }^{4} \mathrm{~F}_{3 / 2}$ & & 4.26116 & 4.26018 & 4.44057 & 4.2396 & $54(13)$ \\
\hline 14 & $3 s^{2} 3 p^{4}\left({ }^{1} D\right) 3 d$ & ${ }^{2} \mathrm{D}_{3 / 2}$ & & 4.31494 & 4.31263 & 4.18973 & 4.2974 & $40(14), 24(31)$ \\
\hline 15 & $3 s^{2} 3 p^{4}\left({ }^{3} P\right) 3 d$ & ${ }^{4} \mathrm{P}_{1 / 2}$ & & 4.31630 & 4.31493 & 4.22252 & 4.3014 & $94(15)$ \\
\hline 16 & $3 s^{2} 3 p^{4}\left({ }^{3} P\right) 3 d$ & ${ }^{4} \mathrm{P}_{3 / 2}$ & & 4.37501 & 4.37375 & 4.23761 & 4.3550 & $72(16)$ \\
\hline 17 & $3 s^{2} 3 p^{4}\left({ }^{3} P\right) 3 d$ & ${ }^{2} \mathrm{~F}_{7 / 2}$ & & 4.39185 & 4.38870 & 4.56722 & & $49(17), 30(21)$ \\
\hline 18 & $3 s^{2} 3 p^{4}\left({ }^{3} P\right) 3 d$ & ${ }^{4} \mathrm{P}_{5 / 2}$ & & 4.39332 & 4.39112 & 4.24533 & 4.3752 & $56(18)$ \\
\hline 19 & $3 s^{2} 3 p^{4}\left({ }^{1} D\right) 3 d$ & ${ }^{2} \mathrm{D}_{5 / 2}$ & & 4.42222 & 4.42012 & 4.30049 & 4.3982 & $37(18), 35(19)$ \\
\hline 20 & $3 \mathrm{~s}^{2} 3 \mathrm{p}^{4}\left({ }^{1} \mathrm{D}\right) 3 \mathrm{~d}$ & ${ }^{2} \mathrm{G}_{9 / 2}$ & & 4.50193 & 4.49828 & 4.67757 & & $91(20)$ \\
\hline 21 & $3 s^{2} 3 p^{4}\left({ }^{1} D\right) 3 d$ & ${ }^{2} \mathrm{G}_{7 / 2}$ & & 4.50445 & 4.50097 & 4.68229 & & $61(21), 25(17)$ \\
\hline 22 & $3 s^{2} 3 p^{4}\left({ }^{3} P\right) 3 d$ & ${ }^{2} \mathrm{~F}_{5 / 2}$ & & 4.53745 & 4.53437 & 4.37312 & 4.5042 & $71(22), 20(23)$ \\
\hline 23 & $3 s^{2} 3 p^{4}\left({ }^{1} D\right) 3 d$ & ${ }^{2} \mathrm{~F}_{5 / 2}$ & & 4.81615 & 4.81085 & 4.65453 & 4.7808 & $75(23), 17(22)$ \\
\hline 24 & $3 \mathrm{~s}^{2} 3 \mathrm{p}^{4}\left({ }^{1} \mathrm{D}\right) 3 \mathrm{~d}$ & ${ }^{2} \mathrm{~F}_{7 / 2}$ & & 4.86106 & 4.85571 & 5.02601 & & $77(24), 17(17)$ \\
\hline 25 & $3 s^{2} 3 p^{4}\left({ }^{1} S\right) 3 d$ & ${ }^{2} \mathrm{D}_{3 / 2}$ & & 5.09978 & 5.09465 & 4.98016 & 5.0663 & $66(25), 25(14)$ \\
\hline 26 & $3 s^{2} 3 p^{4}\left({ }^{1} S\right) 3 d$ & ${ }^{2} \mathrm{D}_{5 / 2}$ & & 5.15048 & 5.14533 & 5.01961 & 5.1158 & $72(26), 17(19)$ \\
\hline 27 & $3 s^{2} 3 p^{4}\left({ }^{1} D\right) 3 d$ & ${ }^{2} \mathrm{~S}_{1 / 2}$ & 5.30822 & 5.41875 & 5.40132 & 5.31352 & 5.4026 & $69(27), 24(3)$ \\
\hline 28 & $3 s^{2} 3 p^{4}\left({ }^{3} P\right) 3 d$ & ${ }^{2} \mathrm{P}_{3 / 2}$ & 5.52611 & 5.65882 & 5.64209 & 4.14934 & 5.6123 & $50(28), 39(12)$ \\
\hline 29 & $3 s^{2} 3 p^{4}\left({ }^{3} P\right) 3 d$ & ${ }^{2} \mathrm{P}_{1 / 2}$ & 5.59044 & 5.72285 & 5.70565 & 4.06195 & 5.6745 & $51(29), 43(9)$ \\
\hline 30 & $3 s^{2} 3 p^{4}\left({ }^{3} P\right) 3 d$ & ${ }^{2} \mathrm{D}_{5 / 2}$ & 5.60557 & 5.73908 & 5.72053 & 5.60460 & 5.7048 & $66(30), 20(19)$ \\
\hline 31 & $3 s^{2} 3 p^{4}\left({ }^{3} P\right) 3 d$ & ${ }^{2} \mathrm{D}_{3 / 2}$ & 5.75629 & 5.88839 & 5.86979 & 5.75540 & 5.8427 & $60(31), 16(14)$ \\
\hline
\end{tabular}

NIST: http://physics.nist.gov/PhysRefData.

GRASP: present results from the GRASP code with 2608 levels.

FAC: present results from the FAC code with 2608 levels.

CIV3: results of Verma et al. (2005) from the cIV3 code.

MCHF: unpublished results of Irimia \& Froese-Fischer (2003) available at http://www.vuse.vanderbilt.edu/ cff/mchf_collection/.

${ }^{a}$ Numbers of the form $M(N)$ mean that it is $M \%$ of the level number $N$.

contributions listed in Table $2 \mathrm{~b}$. Since the levels are (generally) identified on the basis of the strength of their eigenvectors, we see no ambiguity in their identification. Apart from this, we have performed a series of test calculations with differing amount of $\mathrm{CI}$, as discussed above with respect to Table $2 \mathrm{a}$, and in each instance we find the same orderings. Interestingly, the mixing coefficients for the $3 \mathrm{~s}^{2} 3 \mathrm{p}^{4}\left({ }^{3} \mathrm{P}\right) 3 \mathrm{~d}^{2} \mathrm{P}_{3 / 2}$ level in our GRASP and their CIV3 calculations are also similar, yet their level identification is different. Since they have not provided mixing coefficients for other levels, we cannot comment any further on their level orderings. However, we would like to emphasize here that it is not always easy to identify the levels, as the eigenvector from the same level/configuration may dominate for two (or even more) levels, as may be noted for levels 18 and 19 , i.e. $3 s^{2} 3 p^{4}\left({ }^{3} \mathrm{P}\right) 3 \mathrm{~d}$ ${ }^{4} \mathrm{P}_{5 / 2}$ and $3 \mathrm{~s}^{2} 3 \mathrm{p}^{4}\left({ }^{1} \mathrm{D}\right) 3 \mathrm{~d}^{2} \mathrm{D}_{5 / 2}$. This is a common problem in all atomic structure calculations, especially when the levels of the same $J$ value either from the same or different configuration(s) are highly mixed, and the addition of more CI complicates the identifications further rather than making the task easier see Aggarwal \& Keenan (2007) for further details and comparisons. Therefore, the configuration and the $J$ values given in Table 1 are definite, but the corresponding LSJ designation provided for a level is only for guidance, and is liable to interchange.
Additionally, for highly mixed levels the $L S J$ designations provided in Table 1 are not fully appropriate and the $j j$ coupling scheme, as adopted in the calculations, is more suitable. Therefore, in Table 1 we have also provided the corresponding $j j$ designations to facilitate the level/configuration identifications.

Since Verma et al. (2005) have also listed energies for the levels of the $\left(3 s^{2} 3 p^{4}\right) 4 s$ and $4 d$ configurations, in Table $2 c$ we compare our results from the GRASP calculations. The corresponding results from the FAC calculations are not listed here because they are very similar as already discussed. Also listed in this table are the experimental compilations from NIST, which are available for only a few levels. For the levels for which Verma et al. have adjusted their Hamiltonian, their energy levels are in complete agreement with those of NIST, and our results are invariably higher by $\sim 0.3 \mathrm{Ryd}(\leq 3 \%)$. For most of the remaining levels the results of Verma et al. are higher than ours by $\sim 0.4$ Ryd (3\%), and more importantly their orderings do not agree with our work, as in case of the lowest 31 levels of Table $2 \mathrm{a}$. As stated earlier, we have performed a series of calculations from both GRASP and FAC codes, and in each instance we obtain the same orderings as listed here in Table 2c. Therefore, based on this comparison and the discussions above, we may conclude that the level orderings of the Verma et al. calculations 
Table 2. c) Comparison of energy levels (in Ryd) from the $\left(3 s^{2} 3 p^{4}\right) 4 s$ and $4 d$ configurations of Co XI.

\begin{tabular}{|c|c|c|c|c|c|c|}
\hline Index & Configuration & Level & NIST & GRASP $^{a}$ & GRASP $^{b}$ & CIV3 \\
\hline 213 & $3 s^{2} 3 p^{4}\left({ }^{3} P\right) 4 s$ & ${ }^{4} \mathrm{P}_{5 / 2}$ & 10.76298 & 11.04757 & 11.03993 & 10.76940 \\
\hline 224 & $3 s^{2} 3 p^{4}\left({ }^{3} P\right) 4 s$ & ${ }^{4} \mathrm{P}_{3 / 2}$ & 10.84335 & 11.13175 & 11.12353 & 10.84154 \\
\hline 232 & $3 s^{2} 3 p^{4}\left({ }^{3} P\right) 4 s$ & ${ }^{4} \mathrm{P}_{1 / 2}$ & & 11.20260 & 11.19201 & 11.23417 \\
\hline 235 & $3 s^{2} 3 p^{4}\left({ }^{3} P\right) 4 s$ & ${ }^{2} \mathrm{P}_{3 / 2}$ & 10.95407 & 11.25392 & 11.24456 & 10.95744 \\
\hline 241 & $3 s^{2} 3 p^{4}\left({ }^{3} P\right) 4 s$ & ${ }^{2} \mathrm{P}_{1 / 2}$ & 11.04255 & 11.35122 & 11.34096 & 11.04213 \\
\hline 246 & $3 s^{2} 3 p^{4}\left({ }^{1} D\right) 4 s$ & ${ }^{2} \mathrm{D}_{5 / 2}$ & 11.18024 & 11.50776 & 11.49737 & 11.18159 \\
\hline 247 & $3 s^{2} 3 p^{4}\left({ }^{1} D\right) 4 s$ & $\begin{array}{l}{ }^{2} \mathrm{D}_{3 / 2} \\
\end{array}$ & 11.18772 & 11.51474 & 11.50419 & 11.19064 \\
\hline 287 & $3 s^{2} 3 p^{4}\left({ }^{1} S\right) 4 s$ & ${ }^{2} \mathrm{~S}_{1 / 2}$ & & 12.00011 & 11.98931 & 11.72369 \\
\hline 475 & $3 s^{2} 3 p^{4}\left({ }^{3} P\right) 4 d$ & ${ }^{4} \mathrm{D}_{7 / 2}$ & & 13.60829 & 13.59759 & 13.89240 \\
\hline 476 & $3 s^{2} 3 p^{4}\left({ }^{3} P\right) 4 d$ & $\begin{array}{l}{ }^{4} \mathrm{D}_{5 / 2} \\
\end{array}$ & & 13.61116 & 13.59983 & 13.94405 \\
\hline 477 & $3 s^{2} 3 p^{4}\left({ }^{3} P\right) 4 d$ & $\begin{array}{l}{ }^{4} \mathrm{D}_{3 / 2} \\
\end{array}$ & & 13.62434 & 13.61456 & 14.01072 \\
\hline 481 & $3 s^{2} 3 p^{4}\left({ }^{3} P\right) 4 d$ & $\begin{array}{l}{ }^{4} \mathrm{D}_{1 / 2} \\
1 / 2\end{array}$ & & 13.64900 & 13.63838 & 14.03371 \\
\hline 497 & $3 s^{2} 3 p^{4}\left({ }^{3} P\right) 4 d$ & ${ }^{4} \mathrm{~F}_{9 / 2}$ & & 13.70601 & 13.69531 & 13.95476 \\
\hline 499 & $3 s^{2} 3 p^{4}\left({ }^{3} P\right) 4 d$ & ${ }^{2} \mathrm{D}_{5 / 2}$ & 13.40656 & 13.70786 & 13.69708 & 13.40771 \\
\hline 501 & $3 s^{2} 3 p^{4}\left({ }^{3} P\right) 4 d$ & ${ }^{2} \mathrm{~F}_{7 / 2}$ & & 13.71527 & 13.70087 & 13.97201 \\
\hline 503 & $3 s^{2} 3 p^{4}\left({ }^{3} P\right) 4 d$ & ${ }^{2} \mathrm{D}_{3 / 2}$ & & 13.72264 & 13.71180 & 13.63745 \\
\hline 508 & $3 s^{2} 3 p^{4}\left({ }^{3} P\right) 4 d$ & ${ }^{4} \mathrm{P}_{1 / 2}$ & & 13.75090 & 13.74103 & 14.10339 \\
\hline 514 & $3 \mathrm{~s}^{2} 3 \mathrm{p}^{4}\left({ }^{3} \mathrm{P}\right) 4 \mathrm{~d}$ & ${ }^{2} \mathrm{P}_{1 / 2}$ & & 13.80991 & 13.79706 & 13.70203 \\
\hline 518 & $3 s^{2} 3 p^{4}\left({ }^{3} P\right) 4 d$ & ${ }^{4} \mathrm{~F}_{5 / 2}$ & & 13.83298 & 13.81724 & 14.05593 \\
\hline 520 & $3 s^{2} 3 p^{4}\left({ }^{3} P\right) 4 d$ & ${ }^{4} \mathrm{P}_{3 / 2}$ & & 13.83450 & 13.81856 & 14.12163 \\
\hline 521 & $3 s^{2} 3 p^{4}\left({ }^{3} P\right) 4 d$ & $\begin{array}{l}{ }^{1} \mathrm{~F}_{7 / 2}^{3 / 2} \\
\end{array}$ & & 13.83555 & 13.82111 & 14.07921 \\
\hline 524 & $3 s^{2} 3 p^{4}\left({ }^{3} P\right) 4 d$ & ${ }^{4} \mathrm{~F}_{3 / 2}$ & & 13.84944 & 13.83774 & 14.07400 \\
\hline 529 & $3 s^{2} 3 p^{4}\left({ }^{3} P\right) 4 d$ & ${ }^{2} \mathrm{~F}_{5 / 2}$ & & 13.88398 & 13.87033 & 13.77266 \\
\hline 530 & $3 s^{2} 3 p^{4}\left({ }^{3} P\right) 4 d$ & ${ }^{4} \mathrm{P}_{5 / 2}$ & & 13.89370 & 13.87660 & 14.13325 \\
\hline 532 & $3 s^{2} 3 p^{4}\left({ }^{3} P\right) 4 d$ & ${ }^{2} \mathrm{P}_{3 / 2}$ & & 13.90414 & 13.89077 & 13.82993 \\
\hline 543 & $3 \mathrm{~s}^{2} 3 \mathrm{p}^{4}\left({ }^{1} \mathrm{D}\right) 4 \mathrm{~d}$ & ${ }^{2} \mathrm{~S}_{1 / 2}$ & & 13.99888 & 13.98516 & 13.86276 \\
\hline 547 & $3 \mathrm{~s}^{2} 3 \mathrm{p}^{4}\left({ }^{1} \mathrm{D}\right) 4 \mathrm{~d}$ & ${ }^{2} \mathrm{P}_{3 / 2}$ & & 14.03964 & 14.02633 & 13.94990 \\
\hline 550 & $3 \mathrm{~s}^{2} 3 \mathrm{p}^{4}\left({ }^{1} \mathrm{D}\right) 4 \mathrm{~d}$ & ${ }^{2} \mathrm{G}_{7 / 2}$ & & 14.06096 & 14.04660 & 14.30318 \\
\hline 552 & $3 \mathrm{~s}^{2} 3 \mathrm{p}^{4}\left({ }^{1} \mathrm{D}\right) 4 \mathrm{~d}$ & ${ }^{2} \mathrm{G}_{9 / 2}$ & & 14.06988 & 14.05585 & 14.30963 \\
\hline 554 & $3 \mathrm{~s}^{2} 3 \mathrm{p}^{4}\left({ }^{1} \mathrm{D}\right) 4 \mathrm{~d}$ & ${ }^{2} \mathrm{P}_{1 / 2}$ & & 14.07144 & 14.05709 & 13.97781 \\
\hline 564 & $3 \mathrm{~s}^{2} 3 \mathrm{p}^{4}\left({ }^{1} \mathrm{D}\right) 4 \mathrm{~d}$ & ${ }^{2} \mathrm{D}_{5 / 2}$ & 13.76742 & 14.11511 & 14.10145 & 13.77487 \\
\hline 566 & $3 \mathrm{~s}^{2} 3 \mathrm{p}^{4}\left({ }^{1} \mathrm{D}\right) 4 \mathrm{~d}$ & $\begin{array}{l}{ }^{2} \mathrm{D}_{3 / 2} \\
\end{array}$ & 13.88224 & 14.13103 & 14.11527 & 13.86195 \\
\hline 575 & $3 \mathrm{~s}^{2} 3 \mathrm{p}^{4}\left({ }^{1} \mathrm{D}\right) 4 \mathrm{~d}$ & ${ }^{2} \mathrm{~F}_{5 / 2}$ & & 14.18792 & 14.17540 & 14.10631 \\
\hline 579 & $3 \mathrm{~s}^{2} 3 \mathrm{p}^{4}\left({ }^{1} \mathrm{D}\right) 4 \mathrm{~d}$ & ${ }^{2} \mathrm{~F}_{7 / 2}$ & & 14.19597 & 14.18295 & 14.43582 \\
\hline 629 & $3 \mathrm{~s}^{2} 3 \mathrm{p}^{4}\left({ }^{1} \mathrm{~S}\right) 4 \mathrm{~d}$ & ${ }^{2} \mathrm{D}_{3 / 2}$ & & 14.62744 & 14.61441 & \\
\hline 630 & $3 s^{2} 3 p^{4}\left({ }^{1} S\right) 4 d$ & ${ }^{2} \mathrm{D}_{5 / 2}$ & & 14.63222 & 14.61842 & \\
\hline
\end{tabular}

NIST: http://physics.nist.gov/PhysRefData.

GRASP: present results from the GRASP code without (a) and with (b) the inclusion of Breit and QED energies with 2608 levels. CIV3: results of Verma et al. (2005) from the cIV3 code.

do not agree with any other theoretical work. Furthermore, their energy levels have scope for improvement, not because of lack of $\mathrm{CI}$ but due to the exclusion of two-body relativistic operators, as demonstrated and discussed in detail by Aggarwal et al. (2007). Finally, based on a series of calculations and various comparisons, our energy levels are assessed to be accurate to better than $3 \%$ for a majority of levels.

\section{Radiative rates}

The absorption oscillator strength $\left(f_{i j}\right)$ and radiative rate $A_{j i}$ (in $\mathrm{s}^{-1}$ ) for a transition $i \rightarrow j$ are related by the following expression:

$f_{i j}=\frac{m c}{8 \pi^{2} e^{2}} \lambda_{j i}^{2} \frac{\omega_{j}}{\omega_{i}} A_{j i}=1.49 \times 10^{-16} \lambda_{j i}^{2}\left(\omega_{j} / \omega_{i}\right) A_{j i}$

where $m$ and $e$ are the electron mass and charge, respectively, $c$ is the velocity of light, $\lambda_{j i}$ is the transition energy/wavelength in $\AA$, and $\omega_{i}$ and $\omega_{j}$ are the statistical weights of the lower $(i)$ and upper $(j)$ levels, respectively. Similarly, the oscillator strength $f_{i j}$ (dimensionless) and the line strength $\mathrm{S}$ (in atomic unit, 1 a.u. = $6.460 \times 10^{-36} \mathrm{~cm}^{2} \mathrm{esu}^{2}$ ) are related by the following standard equations:

For the electric dipole (E1) transitions:

$A_{j i}=\frac{2.0261 \times 10^{18}}{\omega_{j} \lambda_{j i}^{3}} S^{\mathrm{E} 1} \quad$ and $\quad f_{i j}=\frac{303.75}{\lambda_{j i} \omega_{i}} S^{\mathrm{E} 1}$,

for the magnetic dipole (M1) transitions:

$A_{j i}=\frac{2.6974 \times 10^{13}}{\omega_{j} \lambda_{j i}^{3}} S^{\mathrm{M} 1} \quad$ and $\quad f_{i j}=\frac{4.044 \times 10^{-3}}{\lambda_{j i} \omega_{i}} S^{\mathrm{M} 1}$,

for the electric quadrupole (E2) transitions:

$A_{j i}=\frac{1.1199 \times 10^{18}}{\omega_{j} \lambda_{j i}^{5}} S^{\mathrm{E} 2} \quad$ and $\quad f_{i j}=\frac{167.89}{\lambda_{j i}^{3} \omega_{i}} S^{\mathrm{E} 2}$,

and for the magnetic quadrupole (M2) transitions:

$A_{j i}=\frac{1.4910 \times 10^{13}}{\omega_{j} \lambda_{j i}^{5}} S^{\mathrm{M} 2} \quad$ and $\quad f_{i j}=\frac{2.236 \times 10^{-3}}{\lambda_{j i}^{3} \omega_{i}} S^{\mathrm{M} 2}$ 
In Table 3 we present transition energies/wavelengths ( $\lambda$ in $\AA$ ), radiative rates $\left(A_{j i}\right.$ in $\left.\mathrm{s}^{-1}\right)$ and oscillator strengths $\left(f_{i j}\right.$, dimensionless), in length form only, for all (10422) electric dipole (E1) transitions with $f$-values $\geq 10^{-5}$ among the lowest $287 \mathrm{lev}$ els of Co XI. The indices used to represent the lower and upper levels of a transition have already been defined in Table 1. Similarly, there are 15520 electric quadrupole (E2), 10884 magnetic dipole (M1) and 14796 magnetic quadrupole (M2) transitions among the lowest 287 levels. However, the $f$-values for a majority of these forbidden transitions are very small, and therefore in Tables 4 and 5 we list the $A$ - and $f$-values of only those E2 and M1 transitions, whose $f$-values are $\geq 10^{-8}$. Finally, in Table 6 we list the $A$ - and $f$-values of only those M2 transitions, whose $f$-values are $\geq 10^{-10}$. However, a complete set of $A$ - and $f$-values for all transitions can be obtained on request from KMA (K.Aggarwal@qub.ac.uk). Furthermore, the corresponding $S$ values for any type of transition can be easily obtained by using Eqs. (2)-(5).

Since the E1 transitions are comparatively more important, and have larger oscillator strengths, we focus on the accuracy assessment of these transitions alone. In Table 7 we compare our $f$-values from the GRASP1, GRASP2, GRASP3, FAC1 and FAC2 calculations with the available results of Verma et al. (2005) from the CIV3 code and of Irimia \& Froese-Fischer (2003) from the MCHF code. Also included in this table are the ratio of the length and velocity forms of the $f$-values from the GRASP3 calculations. In general, our calculations from the GRASP code with differing amount of CI agree within $\sim 10 \%$. However, differences for some weak transitions, such as 1-247 and $2-13$, are up to a factor of three, whereas for the 2-6 transition $\left(f \sim 10^{-7}\right)$ the $f$-values differ by up to an order of magnitude. The weak transitions are very sensitive to mixing coefficients, and hence differing amount of CI provide different results. However, such transitions do not affect the overall accuracy of the calculations. Among the stronger transitions, the GRASP3 calculations yield $40 \%$ higher results than from GRASP2 for the $2-247\left(3 \mathrm{~s}^{2} 3 \mathrm{p}^{5}{ }^{2} \mathrm{P}_{1 / 2}^{\circ}-3 \mathrm{~s}^{2} 3 \mathrm{p}^{4} 4 \mathrm{~s}^{2} \mathrm{D}_{3 / 2}\right)$ transition, which reflects the inadequate CI included in GRASP2. This can be confirmed by a comparison with similar calculations performed from the FAC code, with an increased CI in FAC2. Not only the 2-247 but (almost) all strong transitions demonstrate an insignificant effect of including the additional CI in the FAC2 calculations. Similarly, the MCHF results of Irimia \& FroeseFischer compare well with our GRASP and FAC calculations for all strong transitions, and differences for the weaker ones are also within a factor of two. On the other hand, the CIV3 results of Verma et al. only agree with the other calculations for the strong transitions. For weaker transitions, their $f$-values are incompatible with other calculations, and differences of an order of magnitude are notable for many, such as: $1-7,1-16,2-7$ and 2-16, and for the very weak $2-6$ transition, their $f$-value is higher by over three orders of magnitude. Although weak transitions are comparatively less important and are less reliable for accuracy assessment, a significant difference with all other calculations indicates a comparatively lesser accuracy for their results, in spite of the fact that their wavelengths for some transitions may be more accurate, because of the adjustment with corresponding experimental values, as already discussed in Sect. 2 . This is because the basic errors in computing the matrix elements for the determination of $A$-values (and other related parameters) still remain and are not corrected by the adjustment procedure (Hibbert 1975b). However, since they have reported only limited results which are inadequate for applications in plasma modelling, we do not discuss these any further.
In the presence of the limited comparison shown for the $f$ values in Table 7 , it is difficult to assess the accuracy of our present results. A general criterion to assess the accuracy is to compare the length and velocity forms of the $f$ - or $A$-values. However, before we discuss these we remind readers that such comparisons are only desirable, but are not a fully sufficient test to assess accuracy, as different calculations (or combinations of configurations) may give comparable $f$-values in the two forms, but entirely different results in magnitude. This is because in the dipole length form relativistic corrections are automatically included, but extra terms should be added to the gradient matrix element to restore the equivalence between the length and velocity formalisms. In cases where the $L S$ coupling scheme is sufficiently good, the velocity form (uncorrected gradient) could still be used for allowed transitions, but for spin-forbidden transitions, the velocity form is unsuitable (Hibbert 1975b). Generally, there is a good agreement between the length and velocity forms of the $f$-values for strong transitions, as already shown for some in Table 7. However, differences between the two forms can be substantial even for some very strong transitions, as discussed in detail by Aggarwal et al. (2007).

Since the electric dipole (E1) transitions are comparatively more important, we focus solely on the accuracy assessments of their $A$-values. Among the stronger transitions (i.e. $f \geq 0.01$ ), the length and velocity forms differ by over $20 \%$ for only 174 (1.7\%) transitions. However, for 26 transitions the two forms differ by over $50 \%$, but lie within a factor of four. Therefore, based on a satisfactory agreement between the two forms, we may state that the accuracy of our listed $A$-values is better than $20 \%$ for a majority of the strong transitions. Differences between the two forms for weaker transitions $(f<0.01)$ can sometimes be several orders of magnitude, see for example transition 2-6 in Table 7. However, for $118(\sim 1 \%)$ transitions, the ratio $A_{\mathrm{L}} / A_{\mathrm{V}}$ or $f_{\mathrm{L}} / f_{\mathrm{V}}$ is very large. Examples are: $39-283\left(f=4.7 \times 10^{-10}\right)$, $160-247\left(f=2.9 \times 10^{-11}\right), 197-200\left(f=3.4 \times 10^{-8}\right), 215-216$ $\left(f=1.1 \times 10^{-10}\right)$, and $268-280\left(f=1.2 \times 10^{-14}\right)$. All such transitions are very weak, and hence sensitive to mixing coefficients, as already discussed above, but do not affect the overall accuracy of the calculations. However, before concluding we make one more comparison with our calculations from FAC.

A comparison between the $A$-values from GRASP and FAC (not listed in Table 3 ) indicates excellent agreement for a majority of the strong transitions, as only $75(\leq 1 \%)$ have $f$-values differing by over $20 \%$. Considering that the calculations have been performed for a large number of transitions, such differences for a few transitions do not affect the overall accuracy of our results, and we may state with confidence that our listed $A$-values are probably accurate to better than $20 \%$ for a majority of the strong transitions.

\section{Lifetimes}

The lifetime $\tau$ for a level $j$ is defined as follows:

$\tau_{j}=\frac{1}{\sum_{i} A_{j i}}$.

Since this is a measurable parameter, it provides a check on the accuracy of the calculations. Therefore, in Table 1 we have listed our calculated lifetimes, which include the contributions from four types of transitions, i.e. E1, E2, M1 and M2.

In Table 8 we compare our lifetimes from both the GRASP and FAC calculations with the earlier available results of Verma et al. (2005) and Irimia \& Froese-Fischer (2003) for the lowest 
Table 7. Comparison of oscillator strengths ( $f$-values) from the levels of the $3 s^{2} 3 p^{5}$ ground configuration to higher excited levels of Co XI.

\begin{tabular}{|c|c|c|c|c|c|c|c|c|c|}
\hline$I$ & $J$ & GRASP1 & GRASP2 & GRASP3 & $f_{\mathrm{L}} / f_{\mathrm{V}}$ & FAC1 & FAC2 & CIV3 & MCHF \\
\hline 1 & 3 & $2.651-2$ & $2.6471-2$ & $2.636-2$ & 0.89 & $2.713-2$ & $2.677-2$ & $2.599-2$ & $2.865-2$ \\
\hline 1 & 4 & $1.057-4$ & $9.8118-5$ & $9.699-5$ & 1.20 & $9.373-5$ & $9.641-5$ & $4.306-5$ & $6.717-5$ \\
\hline 1 & 6 & $7.850-5$ & $7.1615-5$ & $7.038-5$ & 0.75 & $6.924-5$ & $6.698-5$ & $3.059-4$ & $5.547-5$ \\
\hline 1 & 7 & $2.690-5$ & $2.4571-5$ & $2.399-5$ & 0.59 & $2.562-5$ & $2.458-5$ & $1.712-4$ & $2.093-5$ \\
\hline 1 & 9 & $2.356-4$ & $2.0265-4$ & $1.835-4$ & 0.36 & $1.909-4$ & $1.677-4$ & $2.764-4$ & $1.747-4$ \\
\hline 1 & 11 & $7.046-4$ & $6.8461-4$ & $6.877-4$ & 1.00 & $7.044-4$ & $7.017-4$ & $1.821-3$ & $7.223-4$ \\
\hline 1 & 12 & $1.586-3$ & $1.6535-3$ & $2.027-3$ & 0.60 & $1.771-3$ & $2.439-3$ & $3.283-3$ & $1.605-3$ \\
\hline 1 & 13 & $1.684-3$ & $1.3667-3$ & $8.597-4$ & 0.66 & $1.163-3$ & $3.037-4$ & $5.626-4$ & $5.296-4$ \\
\hline 1 & 14 & $2.113-3$ & $2.1862-3$ & $2.223-3$ & 0.89 & $2.275-3$ & $1.449-3$ & $3.070-4$ & $1.904-3$ \\
\hline 1 & 15 & $1.389-3$ & $1.4124-3$ & $1.398-3$ & 1.00 & $1.440-3$ & $2.209-3$ & $1.190-3$ & $1.148-3$ \\
\hline 1 & 16 & $6.614-5$ & $4.9558-5$ & $4.860-5$ & 0.11 & $5.088-5$ & $4.612-5$ & $2.396-4$ & $2.713-5$ \\
\hline 1 & 18 & $1.481-3$ & $1.6702-3$ & $1.604-3$ & 0.84 & $1.726-3$ & $1.517-3$ & $8.999-4$ & $1.421-3$ \\
\hline 1 & 19 & $7.603-4$ & $8.1102-4$ & $9.650-4$ & 0.75 & $9.502-4$ & $9.641-4$ & $1.342-3$ & $7.232-4$ \\
\hline 1 & 22 & $3.275-4$ & $3.2701-4$ & $3.250-4$ & 0.91 & $3.343-4$ & $3.008-4$ & $9.135-5$ & $2.311-4$ \\
\hline 1 & 23 & $1.880-3$ & $1.8956-3$ & $1.911-3$ & 1.10 & $1.944-3$ & $1.976-3$ & $1.266-3$ & $1.520-3$ \\
\hline 1 & 25 & $4.669-3$ & $4.6165-3$ & $4.513-3$ & 0.91 & $4.663-3$ & $4.212-3$ & $3.210-3$ & $3.599-3$ \\
\hline 1 & 26 & $2.787-3$ & $2.4759-3$ & $2.026-3$ & 1.00 & $2.044-3$ & $1.576-3$ & $6.135-4$ & $1.377-3$ \\
\hline 1 & 27 & $3.065-1$ & $3.1111-1$ & $3.176-1$ & 1.00 & $3.138-1$ & $3.123-1$ & $3.315-1$ & $3.271-1$ \\
\hline 1 & 28 & $6.847-1$ & $6.9195-1$ & $7.000-1$ & 0.96 & $6.917-1$ & $6.959-1$ & $6.691-1$ & $6.914-1$ \\
\hline 1 & 29 & $8.484-2$ & $8.5332-2$ & $8.431-2$ & 0.93 & $8.371-2$ & $8.589-2$ & $7.500-2$ & $7.789-2$ \\
\hline 1 & 30 & $1.201-0$ & $1.2108-0$ & $1.231-0$ & 1.00 & $1.212-0$ & $1.216-0$ & $1.199-0$ & $1.239-0$ \\
\hline 1 & 31 & $2.464-2$ & $2.5584-2$ & $2.830-2$ & 1.10 & $2.719-2$ & $2.516-2$ & $3.094-2$ & $3.385-2$ \\
\hline 1 & 213 & & $4.5024-4$ & $3.846-3$ & 0.92 & $3.594-3$ & $3.677-3$ & $3.348-3$ & \\
\hline 1 & 224 & & $3.8360-2$ & $4.515-2$ & 0.93 & $3.912-2$ & $3.721-2$ & $3.645-2$ & \\
\hline 1 & 232 & & $4.2006-4$ & $4.347-4$ & 0.92 & $4.157-4$ & $4.690-4$ & $1.480-3$ & \\
\hline 1 & 235 & & $1.0720-1$ & $1.157-1$ & 0.93 & $1.165-1$ & $1.148-1$ & $9.650-2$ & \\
\hline 1 & 241 & & $3.4804-2$ & $3.724-2$ & 0.93 & $3.779-2$ & $3.708-2$ & $3.371-2$ & \\
\hline 1 & 246 & & $4.7787-2$ & $8.725-2$ & 0.93 & $8.444-2$ & $6.072-2$ & $8.026-2$ & \\
\hline 1 & 247 & & $3.5847-5$ & $1.153-5$ & 1.10 & $4.663-5$ & $4.413-5$ & $1.297-4$ & \\
\hline 1 & 287 & & $1.1848-2$ & $1.769-2$ & 0.94 & $1.687-2$ & $1.230-2$ & $1.335-2$ & \\
\hline 2 & 3 & $2.591-2$ & $2.5879-2$ & $2.581-2$ & 0.88 & $2.654-2$ & $2.621-2$ & $2.421-2$ & $2.685-2$ \\
\hline 2 & 6 & $9.363-9$ & $8.7174-8$ & $1.270-7$ & 24.00 & $2.277-7$ & $4.973-8$ & $3.391-4$ & $2.921-7$ \\
\hline 2 & 7 & $7.740-5$ & $6.9606-5$ & $6.810-5$ & 0.52 & $6.987-5$ & $6.855-5$ & $7.991-4$ & $5.503-5$ \\
\hline 2 & 9 & $2.309-3$ & $2.1070-3$ & $1.983-3$ & 0.57 & $2.016-3$ & $1.905-3$ & $2.782-3$ & $1.681-3$ \\
\hline 2 & 12 & $2.108-3$ & $2.0525-3$ & $2.334-3$ & 0.78 & $2.147-3$ & $2.499-3$ & $5.729-4$ & $2.118-3$ \\
\hline 2 & 13 & $7.113-4$ & $5.9469-4$ & $2.294-4$ & 0.35 & $4.544-4$ & $5.471-6$ & $1.134-3$ & $1.201-4$ \\
\hline 2 & 14 & $2.089-3$ & $2.2905-3$ & $2.321-3$ & 0.82 & $2.400-3$ & $4.963-4$ & $2.095-3$ & $1.674-3$ \\
\hline 2 & 15 & $5.248-4$ & $5.1322-4$ & $4.829-4$ & 0.94 & $5.027-4$ & $2.172-3$ & $4.349-4$ & $4.254-4$ \\
\hline 2 & 16 & $3.062-5$ & $4.4611-5$ & $6.294-5$ & 1.20 & $5.735-5$ & $6.708-5$ & $4.688-4$ & $4.307-5$ \\
\hline 2 & 25 & $3.884-3$ & $4.3159-3$ & $4.938-3$ & 0.97 & $5.156-3$ & $5.775-3$ & $6.819-3$ & $4.337-3$ \\
\hline 2 & 27 & $2.001-1$ & $2.0172-1$ & $1.998-1$ & 1.00 & $1.985-1$ & $2.027-1$ & $2.023-1$ & $1.937-1$ \\
\hline 2 & 28 & $2.660-2$ & $2.8378-2$ & $3.214-2$ & 0.83 & $3.104-2$ & $2.751-2$ & $3.429-2$ & $3.942-2$ \\
\hline 2 & 29 & $5.256-1$ & $5.3240-1$ & $5.445-1$ & 0.95 & $5.372-1$ & $5.340-1$ & $5.302-1$ & $5.531-1$ \\
\hline 2 & 31 & $1.535-0$ & $1.5454-0$ & $1.566-0$ & 1.00 & $1.542-0$ & $1.550-0$ & $1.516-0$ & $1.568-0$ \\
\hline 2 & 224 & & $2.8017-3$ & $3.403-3$ & 0.94 & $2.671-3$ & $2.514-3$ & $2.534-3$ & \\
\hline 2 & 232 & & $2.6335-3$ & $3.019-3$ & 0.93 & $2.732-3$ & $3.003-3$ & $1.602-3$ & \\
\hline 2 & 235 & & $1.7849-2$ & $1.975-2$ & 0.94 & $1.875-2$ & $1.826-2$ & $1.550-2$ & \\
\hline 2 & 241 & & $8.9298-2$ & $9.480-2$ & 0.93 & $9.461-2$ & $9.241-2$ & $9.373-2$ & \\
\hline 2 & 247 & & $1.0127-1$ & $1.435-1$ & 0.93 & $1.398-1$ & $1.312-1$ & $1.326-1$ & \\
\hline 2 & 287 & & $3.1470-2$ & $4.462-2$ & 0.93 & $4.381-2$ & $3.186-2$ & $3.221-2$ & \\
\hline
\end{tabular}

GRASP1: present results from 1392 level calculations.

GRASP2: present results from 1479 level calculations.

GRASP3: present results from 2608 level calculations.

FAC1: present results from 2608 level calculations.

FAC2: present results from 5709 level calculations.

CIV3: results of Verma et al. (2005) from the cIV3 code.

MCHF: unpublished results of Irimia \& Froese-Fischer (2003) available at http://www.vuse.vanderbilt.edu/ cff/mchf_collection/

31 levels of Co XI. With the exception of the $3 s^{2} 3 p^{4}\left({ }^{3} \mathrm{P}\right) 3 \mathrm{~d}{ }^{4} \mathrm{~F}_{3 / 2}$ level (13), our results from GRASP and FAC agree closely within a few percent. Similarly, with the exception of the $3 s^{2} 3 p^{4}\left({ }^{3} P\right) 3 d$ ${ }^{4} \mathrm{P}_{3 / 2}$ level (16), the earlier results of Irimia \& Froese-Fischer also agree closely for most of the levels, although differences of up to $50 \%$ are notable for a few levels, such as: 4, 19 and 26.
Transitions to these levels are all weak, as seen in Table 3, which implies that the $f$-values are very small. Since $f$-values for weak transitions are very sensitive to mixing coefficients, differences of up to a factor of two are not surprising. However, the corresponding results of Verma et al. show comparatively a larger variation of up to an order of magnitude for some of the levels, 
Table 8. Comparison of lifetimes ( $\tau$ in ns) for the lowest 31 levels of Co XI. $a \pm b \equiv a \times 10^{ \pm b}$.

\begin{tabular}{|c|c|c|c|c|c|c|}
\hline $\bar{J}$ & $\begin{array}{l}\text { Configuration } \\
\text { Con }\end{array}$ & Level & GRASP & $\overline{\text { FAC }}$ & $\overline{\text { CIV3 }}$ & MCHF \\
\hline 2 & $3 s^{2} 3 p^{5}$ & ${ }^{2} \mathrm{P}_{1 / 2}^{\circ}$ & $0.779+7$ & & & \\
\hline 3 & $3 s 3 p^{6}$ & ${ }^{2} \mathrm{~S}_{1 / 2}^{1 / 2}$ & $0.204+0$ & $0.197+0$ & $0.208+0,0.256+0$ & $0.190-0$ \\
\hline 4 & $3 s^{2} 3 p^{4}\left({ }^{3} P\right) 3 d$ & ${ }^{4} \mathrm{D}_{5 / 2}$ & $0.131+3$ & $0.135+3$ & $0.270+3,0.552+2$ & $0.188+3$ \\
\hline 5 & $3 s^{2} 3 p^{4}\left({ }^{3} P\right) 3 d$ & ${ }^{4} \mathrm{D}_{7 / 2}$ & $0.132+8$ & & & \\
\hline 6 & $3 s^{2} 3 p^{4}\left({ }^{3} P\right) 3 d$ & ${ }^{4} \mathrm{D}_{3 / 2}$ & $0.119+3$ & $0.121+3$ & $0.168+2,0.130+2$ & $0.151+3$ \\
\hline 7 & $3 s^{2} 3 p^{4}\left({ }^{3} P\right) 3 d$ & ${ }^{4} \mathrm{D}_{1 / 2}$ & $0.755+2$ & $0.722+2$ & $0.751+1,0.514+1$ & $0.898+2$ \\
\hline 8 & $3 s^{2} 3 p^{4}\left({ }^{3} P\right) 3 d$ & ${ }^{4} \mathrm{~F}_{9 / 2}$ & $0.526+8$ & & & \\
\hline 9 & $3 s^{2} 3 p^{4}\left({ }^{1} D\right) 3 d$ & ${ }^{2} \mathrm{P}_{1 / 2}$ & $0.328+1$ & $0.321+1$ & $0.243+1,0.176+1$ & $0.379+1$ \\
\hline 10 & $3 s^{2} 3 p^{4}\left({ }^{3} P\right) 3 d$ & ${ }^{4} \mathrm{~F}_{7 / 2}$ & $0.358+8$ & & & \\
\hline 11 & $3 s^{2} 3 p^{4}\left({ }^{3} P\right) 3 d$ & ${ }^{4} \mathrm{~F}_{5 / 2}$ & $0.151+2$ & $0.147+2$ & $0.524+1,0.321+1$ & $0.197+2$ \\
\hline 12 & $3 s^{2} 3 p^{4}\left({ }^{1} D\right) 3 d$ & ${ }^{2} \mathrm{P}_{3 / 2}$ & $0.222+1$ & $0.249+1$ & $0.204+1,0$ & $0.268+1$ \\
\hline 13 & $3 s^{2} 3 p^{4}\left({ }^{3} P\right) 3 d$ & ${ }^{4} \mathrm{~F}_{3 / 2}$ & $0.710+1$ & $0.500+1$ & $0.582+1,0.345+1$ & $0.890+1$ \\
\hline 14 & $3 s^{2} 3 p^{4}\left({ }^{1} D\right) 3 d$ & ${ }^{2} \mathrm{D}_{3 / 2}$ & $0.203+1$ & $0.198+1$ & $0.559+1,0$ & $0.252+1$ \\
\hline 15 & $3 s^{2} 3 p^{4}\left({ }^{3} P\right) 3 d$ & ${ }^{4} \mathrm{P}_{1 / 2}$ & $0.206+1$ & $0.200+1$ & $0.251+1$ & $0.250+1$ \\
\hline 16 & $3 s^{2} 3 p^{4}\left({ }^{3} P\right) 3 d$ & ${ }^{4} \mathrm{P}_{3 / 2}$ & $0.838+2$ & $0.842+2$ & $0.152+2,0.143+2$ & $0.139+2$ \\
\hline 17 & $3 s^{2} 3 p^{4}\left({ }^{3} P\right) 3 d$ & ${ }^{2} \mathrm{~F}_{7 / 2}$ & $0.874+7$ & & & \\
\hline 18 & $3 s^{2} 3 p^{4}\left({ }^{3} P\right) 3 d$ & ${ }^{4} \mathrm{P}_{5 / 2}$ & $0.603+1$ & $0.561+1$ & $0.115+2$ & $0.686+1$ \\
\hline 19 & $3 s^{2} 3 p^{4}\left({ }^{1} D\right) 3 d$ & ${ }^{2} \mathrm{D}_{5 / 2}$ & $0.990+1$ & $0.101+2$ & $0.752+1,0.733+1$ & $0.134+2$ \\
\hline 20 & $3 s^{2} 3 p^{4}\left({ }^{1} D\right) 3 d$ & ${ }^{2} \mathrm{G}_{9 / 2}$ & $0.767+7$ & & & \\
\hline 21 & $3 s^{2} 3 p^{4}\left({ }^{1} D\right) 3 d$ & ${ }^{2} \mathrm{G}_{7 / 2}$ & $0.810+7$ & & & \\
\hline 22 & $3 s^{2} 3 p^{4}\left({ }^{3} P\right) 3 d$ & ${ }^{2} \mathrm{~F}_{5 / 2}$ & $0.279+2$ & $0.272+2$ & $0.107+3,0.117+3$ & $0.398+2$ \\
\hline 23 & $3 s^{2} 3 p^{4}\left({ }^{1} D\right) 3 d$ & ${ }^{2} \mathrm{~F}_{5 / 2}$ & $0.421+1$ & $0.415+1$ & $0.681+1,0.422+1$ & $0.538+1$ \\
\hline 24 & $3 s^{2} 3 p^{4}\left({ }^{1} D\right) 3 d$ & ${ }^{2} \mathrm{~F}_{7 / 2}$ & $0.286+7$ & & & \\
\hline 25 & $3 s^{2} 3 p^{4}\left({ }^{1} S\right) 3 d$ & ${ }^{2} \mathrm{D}_{3 / 2}$ & $0.702+0$ & $0.679+0$ & $0.786+$ & $0.861-0$ \\
\hline 26 & $3 s^{2} 3 p^{4}\left({ }^{1} S\right) 3 d$ & ${ }^{2} \mathrm{D}_{5 / 2}$ & $0.347+1$ & & $0.121+$ & $0.518+1$ \\
\hline 27 & $3 s^{2} 3 p^{4}\left({ }^{1} D\right) 3 d$ & ${ }^{2} \mathrm{~S}_{1 / 2}$ & $0.516-2$ & $0.524-2$ & $0.517-2$ & $0.510-2$ \\
\hline 28 & $3 s^{2} 3 p^{4}\left({ }^{3} P\right) 3 d$ & ${ }^{2} \mathrm{P}_{3 / 2}$ & & & $0.596-2$, & $0.557-2$ \\
\hline 29 & $3 s^{2} 3 p^{4}\left({ }^{3} P\right) 3 d$ & ${ }^{2} \mathrm{P}_{1 / 2}$ & $0.559-2$ & & $0.616-2,0.594-2$ & $0.570-2$ \\
\hline 30 & $3 s^{2} 3 p^{4}\left({ }^{3} P\right) 3 d$ & ${ }^{2} \mathrm{D}_{5 / 2}$ & $0.461-2$ & $0.471-2$ & $0.496-2,0.488-2$ & $0.463-2$ \\
\hline 31 & $3 s^{2} 3 p^{4}\left({ }^{3} P\right) 3 d$ & ${ }^{2} \mathrm{D}_{3 / 2}$ & $0.469-2$ & $0.480-2$ & $0.506-2,0.495-2$ & $0.470-2$ \\
\hline
\end{tabular}

GRASP: present results from the GRASP code with 2608 level calculations.

FAC: present results from the FAC code with 2608 level calculations.

CIV3: results of Verma et al. (2005) from the CIV3 code, first entry is in length form and the second in the velocity form.

MCHF: unpublished results of Irimia \& Froese-Fischer (2003) available at http://www.vuse.vanderbilt.edu/ cff/mchf_collection/.

such as: 6 and 7 , i.e. $3 s^{2} 3 p^{4}\left({ }^{3} P\right) 3 d{ }^{4} D_{3 / 2,1 / 2}$. Since these differences in $\tau$ values are a direct consequence of the corresponding differences in the $A$-values, we do not discuss these any further. However, we would like to clarify here that although we have also included the contributions of E2, M1 and M2 transitions, for the levels under consideration their contribution is negligible, and hence the lifetimes reported in Table 8 are basically derived from the E1 transitions alone.

The other available results of lifetimes are for the $3 \mathrm{~s} 3 \mathrm{p}^{6}$ ${ }^{2} S_{1 / 2}$ level (3) from Biémont \& Träbert (2000) and Berrington et al. (2001), which are 0.20 and $0.206 \mathrm{~ns}$, respectively. Both of these results agree very well with our $\tau$ value of $0.204 \mathrm{~ns}$ from GRASP. Finally, as stated earlier in Sect. 1, Träbert et al. (2004) have measured the lifetime of the $3 \mathrm{~s}^{2} 3 \mathrm{p}^{5}{ }^{2} \mathrm{P}_{1 / 2}^{\circ}$ level to be $7.62 \pm 0.46 \mathrm{~ms}$. In a later experiment, Träbert et al. (2006) improved the accuracy in their measurement to determine $\tau=$ $7.66 \pm 0.04 \mathrm{~ms}$, which is in excellent agreement with our result of $7.791 \mathrm{~ms}$. Therefore, on the basis of the comparisons shown in Table 8 and discussions above, we may state that in general there is no discrepancy in the determination of the lifetimes for the levels of Co XI. However, additional measurements and/or further calculations, especially for the levels of comparatively higher lifetimes, such as $\left(3 \mathrm{~s}^{2} 3 \mathrm{p}^{4}\left({ }^{3} \mathrm{P}\right) 3 \mathrm{~d}\right){ }^{4} \mathrm{D}_{7 / 2},{ }^{4} \mathrm{~F}_{7 / 2,9 / 2},{ }^{2} \mathrm{~F}_{7 / 2}$, and ${ }^{2} G_{7 / 2,9 / 2}$, will be helpful in further assessing the accuracy of our data.

\section{Conclusions}

In the present work, results for energy levels, radiative rates, and oscillator strengths for all transitions among the lowest $287 \mathrm{lev}-$ els of Co XI have been analysed. Additionally, results for radiative rates have been calculated for four types of transitions, namely E1, E2, M1 and M2. However, the $A$ - and $f$-values have been reported for all E1 transitions with $f \geq 10^{-5}$, but only for the selected $\left(f \geq 10^{-8}\right)$ E2, M1 and M2 transitions. These results for a large range of transitions are likely to be useful for the modelling of a variety of plasmas.

Based on the comparisons made between the length and velocity forms of the oscillator strengths, as well as between the GRASP and FAC calculations, we assess that our radiative rates are accurate to better than $20 \%$ for a majority of the strong E1 transitions. Similarly, based on the comparisons between the GRASP and FAC calculations, as well as with the available compiled experimental results, our energy levels are assessed to be accurate to better than $3 \%$. However, the level designations are likely to fluctuate for a few levels, for which further work may be helpful.

Lifetimes for all excited levels of Co XI are listed, but comparisons have been possible for only the lowest 31 , for which no major discrepancy is observed. 
Acknowledgements. This work has been financed by the Engineering and Physical Sciences and Particle Physics and Astronomy Research Councils of the United Kingdom, and F.P.K. is grateful to AWE Aldermaston for the award of a William Penney Fellowship. A part of this work was performed at the Clark Atlanta University during a visit by KMA in March 2007.

\section{References}

Aggarwal, K. M., \& Keenan, F. P. 2006, A\&A, 450, 1249

Aggarwal, K. M., \& Keenan, F. P. 2007, A\&A, 463, 399

Aggarwal, K. M., Tayal, V., Gupta, G. P., \& Keenan, F. P. 2007, ADNDT, 93, 615

Berrington, K. A., Pelan, J. C., \& Waldock, J. A. 2001, J. Phys. B, 34, L419

Biémont, E, \& Träbert, E. 2000, J. Phys. B, 33, 2939

Del Zanna, G., Berrington, K. A., \& Mason, H. E. 2004, A\&A, 422, 731

Dyall, K. G., Grant, I. P., Johnson, C. T., Parpia, F. A., \& Plummer, E. P. 1989, Comput. Phys. Commun., 55, 424
Fawcett, B. C., \& Hatter, A. T. 1980, A\&A, 84, 78

Fawcett, B. C., \& Hayes, R. W. 1972, J. Phys. B, 5, 366

Fawcett, B. C., Cowan, R. D. \& Hayes, R. W. 1972, J. Phys. B, 5, 2143

Grant, I. P., McKenzie, B. J., Norrington, P. H., Mayers, D. F., \& Pyper, N. C. 1980, Comput. Phys. Commun., 21, 207

Gu, M. F. 2003, ApJ, 582, 1241

Hibbert, A. 1975a, Comput. Phys. Commun., 9, 141

Hibbert, A. 1975b, Rep. Prog. Phys., 38, 1217

Huang, K. N., Kim, Y. K., Cheng, K. T., \& Desclaux, J. P. 1983, ADNDT, 28, 355

Irimia, A., \& Froese-Fischer, C. (2003), available at http://www.vuse.vanderbilt.edu/ cff/mchf_collection/

Liedahl, D. A. 2000, Atomic Data Needs for X-ray Astronomy, available at http://heasarc.gsfc.nasa.gov/docs/heasarc/atomic/

Thomas, R. G., \& Neupert, W. M. 1994, ApJS, 91, 461

Träbert, E., Saathoft, G., \& Wolf, A. 2004, J. Phys. B, 37, 945

Träbert, E., Reinhardt, S., Hoffmann, J., \& Wolf, A. 2006, J. Phys. B, 39, 945

Verma, N., Jha, A. K. S., \& Mohan, M. 2005, J. Phys. B, 38, 3185 